\author{
Dubravko Barisic · Ante Corusic • \\ Mario Coric • Bosko Romic
}

\title{
Diagnosis and treatment of perforated peptic ulcer by emergency laparoscopy in gynecology: a case report
}

Received: 22 October 2005 / Accepted: 10 March 2006 / Published online: 12 July 2006

(C) Springer-Verlag Berlin / Heidelberg 2006

\begin{abstract}
We report on a patient who presented with symptoms of acute abdomen and sudden abdominal pain that had started $4 \mathrm{~h}$ before hospitalization. Emergent laparoscopy was immediately done. A perforated peptic ulcer in the anterior prepyloric region was detected and laparoscopically sutured. The patient's postoperative course was uneventful. The risks and advantages of laparoscopic treatment of peptic ulcer perforation are further discussed.
\end{abstract}

Keywords Laparoscopy $\cdot$ Peptic ulcer $\cdot$ Perforated

\section{Introduction}

Acute abdomen is an emergency situation that can be caused by various conditions, including extrauterine pregnancy, adnexal torsion, hemorrhagic ovarian cyst, appendicitis, and the rupture of different visceral organs. Symptoms and clinical signs are not absolutely specific for each type of above-mentioned pathology. Therefore, the professional responsibility of gynecologists and abdominal surgeons is often overlapping as well as shared.

Perforation of peptic ulcer is one of the causes of acute abdomen in which early diagnosis and treatment dramatically decrease morbidity and mortality [1]. In contrast to gynecologists, abdominal surgeons are familiar with both the diagnostic and the treatment modalities of this condition. Having recently encountered a situation in which a gynecologist detected a perforated peptic ulcer during emergency laparoscopy in a patient without previous history or symptoms of peptic ulcer, and in which the

\footnotetext{
D. Barisic · A. Corusic ·

M. Coric $(\square) \cdot$ B. Romic

Department of Obstetrics and Gynecology,

Zagreb University Hospital Center,

Petrova 13,

10000 Zagreb, Croatia

e-mail: mario.coric@zg.t-com.hr

Tel.: +385-1-4604616

Fax: $+385-1-4633512$
}

same gynecologist was obliged to treat the patient by himself due to delayed availability of an abdominal surgeon, we concluded that gynecologists should become more familiar with the treatment modalities of perforated peptic ulcer.

\section{Case report}

A 52-year-old patient was admitted in the emergency room of the department of obstetrics and gynecology at 9 a.m. because of prostration and acute abdominal pain that had begun suddenly at 5 a.m. the same morning. Personal history revealed appendectomy and no symptoms of peptic ulcer in anamnesis. Gynecological anamnesis revealed an irregular menstrual cycle for the previous 6 months as well as a functional cyst detected on gynecological examination 1 week before.

Physical examination detected guarding of the abdominal wall. Gynecological examination revealed no palpable adnexal masses, although detailed bimanual palpation was impossible because of permanent pain and evident abdominal guarding. Blood analysis revealed normal red blood cells but leukocytosis of $15,000 / \mu \mathrm{l}$. Transaminases as well as amylases were within normal limits. A diagnosis of acute abdomen was made, and an emergency laparoscopy using standard endoscopic equipment was done within 15 min of admission.

Laparoscopy was technically performed using standard endoscopic equipment. On laparoscopy no pathology of the uterus or adnexa was detected, only yellowish-white homogeneous fluid in the pouch of Douglas. The same fluid was detected in both paracolic gutters as well as subdiaphragmatically. A gastric perforation $<10 \mathrm{~mm}$ in diameter in the anterior prepyloric area was detected (Fig. 1). The perforation was sutured using resorbable polydioxanone suturing material for open surgery ( 0 Vicryl on a CT curved needle; Ethicon, Edinburgh, UK). Three sutures, "far-near/near-far," were used to repair the perforation. One 5-mm needle holder (Johnson \& Johnson) was introduced through the left suprapubic trocar, and another 
was introduced in the medial line caudally to the xyphoid bone. Sutures were tied intracorporally (Fig. 2). The abdominal cavity was copiously irrigated and postoperatively drained for $48 \mathrm{~h}$. A nasogastric probe was immediately introduced, as were intravenous cefuroxime $1.5 \mathrm{~g}$ three times daily and intravenous ranitidine $50 \mathrm{mg}$ every $6 \mathrm{~h}$. The procedure was performed in $65 \mathrm{~min}$. The patient's postoperative curse was uneventful, and she was released on the 5 th postoperative day.

\section{Discussion}

The symptoms and clinical signs of perforated gastric or duodenal ulcer are not absolutely specific. The patient may have a history of peptic ulcer disease, but in about one-third of cases, the first symptom is a sudden attack of severe abdominal pain, usually epigastric, as well as reflected pain in another part of the abdomen. An upright abdominal xray shows air under one or both sides of the diaphragm in only half of cases [2]. The incidence of peptic ulcer disease has been estimated as 1,500-3,000 per 100,000 individuals, with the prevalence of perforation estimated to be $5 \%$ per year. Because $3-10$ patients per 100,000 inhabitants [3] are operated on due to peptic ulcer perforation, plus the fact that signs and symptoms can be nonspecific, gynecologists should become more familiar with the treatment modalities of perforated peptic ulcer.

Perforated gastric or duodenal ulcer is one of the most serious types of peritonitis, with mortality as high as $20 \%$ [2]. There is ongoing debate concerning whether perforated peptic ulcers generally need to be operated on. It has been estimated that about half of the perforations seal by themselves [4]. In cases in which the patient has little discomfort and is hemodynamically stable, conservative Taylor's treatment can be tried under a surgeon's strict clinical surveillance [5]. If the abdominal tenderness increases, the patient becomes hemodynamically unstable, or the contrast medium flows freely into the abdomen, then an operation is indicated. Operation in conservatively treated patients proceeds in $10 \%$ [6]. However, early diagnosis and treatment dramatically decrease morbidity and mortality as well. It has been shown that delaying the

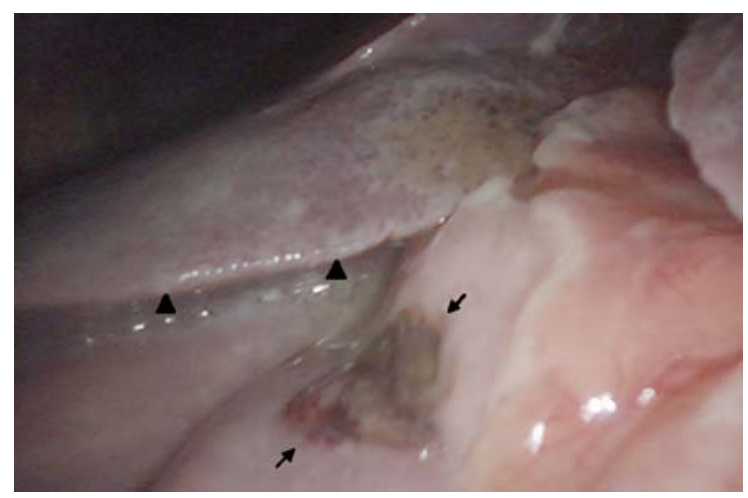

Fig. 1 Laparoscopic view of perforated peptic ulcer (arrows) in the anterior prepyloric area; triangles indicate liver

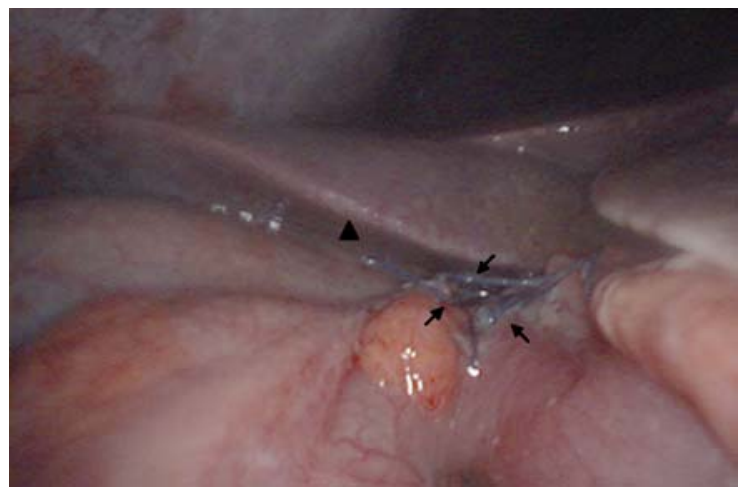

Fig. 2 Suture-repaired site of the perforation (arrows); triangle indicates liver

time of operation beyond $12 \mathrm{~h}$ after the onset of clinical symptoms worsens the outcome [1, 4], as do shock on admission, renal insufficiency, concurrent medical illness, liver cirrhosis, or an immunocompromised state. In those situations the risk increases to almost $30 \%$ [7]. In cases in which the patient presents more than $24 \mathrm{~h}$ after the onset of symptoms or shows the clinical signs of acute abdomen, hypotension, or sepsis, surgery should be done immediately $[4,6]$. Patients 70 years or older are less likely to respond to conservative treatment and might profit from an early decision for surgery [6].

There is currently a trend to avoid a radical approach to perforated peptic ulcer, especially in emergency cases. The two main goals of the surgical procedure for perforated peptic ulcer are to close the perforation and treat the peritonitis [8]. By adopting a conservative treatment policy and extending the basic principles of minimally invasive or maximally sparing surgery, physicians can effectively use the laparoscopic approach to identify the perforation site when it has not been covered by the peritoneal inflammatory reaction. This approach provides an adequate evaluation of the extent of peritonitis and allows treatment of both conditions at the same time. Keeping in mind success of the treatment and unexpected diagnostic problems during gynecological laparoscopy, we believe gynecologists should be familiar with treatment of perforated peptic ulcer.

Many studies have found advantages and less morbidity and mortality after laparoscopic treatment compared with open operation. Laparoscopy is less likely than laparotomy to cause transient translocation of aerobic and anaerobic species, endotoxemia, and development of intraperitoneal abscesses $[9,10]$. Many authors have reported the absence of hernias and evisceration and better postoperative patient comfort reflected by a lower need for analgesics $[8,11]$, but there was no difference in terms of duration of nasogastric aspiration, intravenous drip, hospital stay, or time to resumption of diet. Because of peritonitis in all the patients, gastrointestinal motility recovered in the same period, regardless of the mode of access [9]. Theoretically, the laparoscopic approach causes less metabolic and physiological disturbance than open surgery, which may benefit septic patients. The main disadvantage of laparoscopic repair of perforated peptic ulcer is the significantly longer 
operating time, but this also depends on the surgeon's laparoscopic suturing skills. However, most of the time is spent cleaning up spillage in the abdominal cavity with the irrigation-aspiration cannula.

We performed a traditional "far-near/near-far" suture closing technique for the gastric perforation, which was located in the anterior prepyloric area and was $<1 \mathrm{~cm}$. Some authors believe that peptic ulcers with perforations $>1 \mathrm{~cm}$ cannot be adequately repaired using laparoscopic suturing and suggest open surgery [12]. Others suggest, as in open surgery, an omental patch technique that can be easily performed [13]. The omentum is easy to mobilize and has physiologic sealing properties by tissue adhesion and rich vascularization that hastens tissue healing [14]. Laparoscopic sutureless repair with a gelatin sponge plug and fibrin glue [12] or simple lavage relying on spontaneous sealing have been described to be as safe as the suture repair technique and also reduced operative time. In all techniques mentioned above, the main problem could be the existence of undetected malignancies. To resolve that problem and even help in the operative procedure, especially in filling the defect with omental tissue and stabilizing the omentum under direct vision, some authors suggest a combined intraoperative laparoscopic-gastroendoscopic method [11].

Despite the many advantages of laparoscopy for perforated peptic ulcer that have been reported in the literature, laparoscopy is contraindicated in some situations; laparotomy is preferred in cases of posterior location of the perforation, a very large perforation, or a poor general state of health [15].

Although this one case limits the possibility of drawing definite conclusions, the increase in laparoscopic experience and improvements in technology offer gynecologists a chance to become more familiar with the kind of surgical problem that can sometimes present in atypical form as a "surprise" during emergency laparoscopy. Our professional responsibility often overlaps as well as is shared with that of abdominal surgeons, and today we must try to resolve that kind of surgical problem, especially because we know that early diagnosis and treatment dramatically decrease morbidity and mortality as well.

\section{References}

1. Hermansson M, von Holstein CS, Zilling T (1999) Surgical approach and prognostic factors after peptic ulcer perforation. Eur J Surg 165:566-572

2. Tompkins RG (1992) Peritonitis. In: Berkow R, Fletcher AJ (eds) The Merck manual, 16th edn. Merck, Rahway, NJ, pp 790-794

3. Soll AH (1993) Gastric, duodenal, and stress ulcer. In: Sleisinger MH, Fordtran JS (eds) Gastrointestinal diseases: pathophysiology, diagnosis, management, 5th edn. Saunders, Philadelphia, pp 580-679

4. Donovan AJ, Berne TV, Donovan JA (1998) Perforated duodenal ulcer. An alternative therapeutic plan. Arch Surg 133:1166-1171

5. Taylor H (1946) Perforated peptic ulcer treated without operation. Lancet ii:441-444

6. Marshall C, Ramaswamy P, Bergin FG, Rosenberg IL, Leaper DJ (1999) Evaluation of a protocol for the non-operative management of perforated peptic ulcer. Br J Surg 86:131-134

7. Svanes C, Lie RT, Svanes K, Lie SA, Soreide O (1994) Adverse effects of delayed treatment for perforated peptic ulcer. Ann Surg 220:168-175

8. Johansson B, Hallerback B, Glise H, Johnsson E (1996) Laparoscopic suture closure of perforated peptic ulcer. Surg Endose 10:656-658

9. Michelet I, Agresta F (2000) Perforated peptic ulcer: laparoscopic approach. Eur J Surg 166:405-408

10. Jacobi CA, Ordemann J, Bohm B et al (1997) Does laparoscopy increase bacteremia and endotoxemia in a peritonitis model? Surg Endosc 11:235-238

11. Pescatore P, Halkic N, Calmes JM, Blum A, Gillet M (1998) Combined laparoscopic-endoscopic method using an omental plug for therapy of gastrointestinal ulcer perforation. Gastrointest Endosc 48(4):411-414

12. Lau WY, Leung KL, Kwong KH et al (1996) A randomized study comparing laparoscopic versus open repair of perforated peptic ulcer using suture or sutureless technique. Ann Surg 224 (2):131-138

13. Mouret P, Francois Y, Vignal J, Barth X, Lombard-Platet R (1998) Laparoscopic treatment of perforated peptic ulcer. Br J Surg 77:1006

14. Matoba Y, Katayama H, Ohami H (1996) Evaluation of omental implantation for perforated gastric ulcer therapy: findings in a rat model. J Gastroenterol 31:777-784

15. Zittel TT, Jehle EC, Becker HD (2000) Surgical management of peptic ulcer disease today-indication, technique and outcome. Langebeck's Arch Surg 385:84—96 